\title{
When prediction is fulfilled: Insight from emotion processing
}

\author{
Vicky T. Lai ${ }^{\mathrm{a}, \mathrm{b}, \mathrm{c}, *}$, Falk Huettig a,b \\ a Max Planck Institute for Psycholinguistics, Nijmegen, The Netherlands \\ ${ }^{\mathrm{b}}$ Donders Institute for Brain, Cognition, and Behaviour, Radboud University, Nijmegen, The Netherlands \\ ${ }^{\mathrm{c}}$ Department of Psychology, University of South Carolina, United States
}

\section{A R T I C L E I N F O}

\section{Article history:}

Received 23 July 2015

Received in revised form

1 December 2015

Accepted 13 March 2016

Available online 14 March 2016

Keywords:

Prediction

Emotion

Language

ERP

P200

N400

LPC

\begin{abstract}
A B S T R A C T
Research on prediction in language processing has focused predominantly on the function of predictive context and less on the potential contribution of the predicted word. The present study investigated how meaning that is not immediately prominent in the contents of predictions but is part of the predicted words influences sentence processing. We used emotional meaning to address this question. Participants read emotional and neutral words embedded in highly predictive and non-predictive sentential contexts, with the two sentential contexts rated similarly for their emotional ratings. Event Related Potential (ERP) effects of prediction and emotion both started at $\sim 200 \mathrm{~ms}$. Confirmed predictions elicited larger P200s than violated predictions when the target words were non-emotional (neutral), but such an effect was absent when the target words were emotional. Likewise, emotional words elicited larger P200s than neutral words when the target words were non-predictive, but such effect were absent when the contexts were predictive. We conjecture that the prediction and emotion effects at $\sim 200 \mathrm{~ms}$ may share similar neural process(es). We suggest that such process(es) could be affective, where confirmed predictions and word emotion give rise to 'aha' or rewarding feelings, and/or cognitive, where both prediction and word emotion quickly engage attention.
\end{abstract}

(c) 2016 Elsevier Ltd. All rights reserved.

\section{Introduction}

The extent to which stimuli and the context in which the stimuli occur contribute to processing is of key interest to cognitive sciences. Here we investigated the interaction between words and their preceding context during sentence processing. Numerous studies have examined sentence context driven prediction: Information retrieved from the sentential context can pre-activate aspects of upcoming words prior to their occurrence (e.g., Huettig, 2015, for recent review). Many linguistic features of a predicted target word are activated this way, including semantic/conceptual (Kutas and Federmeier, 2000; Federmeier et al., 2002), conceptualperceptual (Rommers et al., 2013), morpho-syntactic (Van Berkum et al., 2005; Wicha et al., 2003, 2004), phonological (DeLong et al., 2005), and orthographic features (Kim and Lai, 2012; Federmeier and Laszlo, 2009). In contrast, less research has been done with regard to how meaning cued by the target word interacts with context to impact sentence processing.

The current study used Event Related Potentials (ERPs) to investigate the time course of the contributions of word meaning

\footnotetext{
* Corresponding author at: Department of Psychology, University of South Carolina, 1512 Pendleton Street, Columbia, SC 29208, United States.

E-mail address: vicky.tzuyin.lai@gmail.com (V.T. Lai).
}

not immediately prominent or predictable given the sentential context. To this end we used emotionally loaded words, based on the assumption that if any aspect of word meaning were to be activated fast, the emotional aspect would be a good candidate (Zajonc, 2000, but see Storbeck et al., 2006). Emotional words and neutral words were embedded in highly predictive sentential contexts. Critically, the emotional content difference in the contexts between the emotional and neutral conditions was minimized such that the emotional difference became clear only after the emotional and neutral target words appeared. ${ }^{1}$ This design allowed us to test the contribution of the emotional meaning in the predicted word.

Most ERP studies have associated prediction of language meaning with the N400 component. The N400 is a negative deflection occurring in the $300-500 \mathrm{~ms}$ time range post word onset. N400 effects have been found for words that are semantically incongruent with its prior sentential context, relative to those that are congruent (Kutas and Federmeier, 2011). Such effects have

\footnotetext{
${ }^{1}$ It is possible that some emotion associated with the main purport of the prediction (i.e. the target word) can be activated prior to the onset of the predicted word. We dealt with this first by matching the mean emotional ratings of the pretarget contexts between emotional and neutral conditions, and second by focusing on the effects (i.e., subtracting the experimental condition from its control) rather then a given condition in isolation.
} 
been argued to support prediction, as readers obviously cannot predict any semantically incongruent word prior to the onset of those words. However, the very same effect can also be argued to support post-lexical integration. In this case, readers need not form predictions proactively - they could be waiting for the target word before activating the word meaning and integrating the activated word meaning with its context post-lexically. Recent studies demonstrated that, integration or not, the N400 effect is affected by prediction (Van Berkum et al., 2005; DeLong et al., 2005; Kutas et al., 2011). DeLong et al. (2005) examined contextually constraining sentences (e.g. The day was breezy so the boy went outside to fly $a$...) followed by a predicted / semantically congruent word (kite) and an unpredicted / less semantically congruent word (airplane). Not surprisingly, classic N400 effects were found for the unpredicted / incongruent relative to the predicted / congruent words, supporting both prediction and integration accounts. Critically, N400 effects were found for the indefinite article before the unpredicted words relative to the one before the predicted words (a), supporting the prediction account. This suggests that the readers had the predicted word in mind along with its indefinite article before the onset of the target word. A recent study has localized the effects to 350-450 ms (Lau et al., 2014), which very likely represents a time course difference between the N400 effect elicited by semantic violation and the N400 effect elicited by prediction violation.

Several language prediction studies have also reported modulations of the Late Positive Component (LPC). Low-predictive words elicited more positive LPCs than high-predictive ones (Van Petten and Luka, 2012; DeLong et al., 2014). The LPCs in these studies have a less distinct time frame, and tend to occur at $\sim 600 \mathrm{~ms}$. The scalp distributions of the prediction LPC effects can be posterior or anterior, depending on the plausibility of the target words given their contexts (DeLong et al., 2014). When the target word is not plausible (i.e., anomalous), the LPC effect for lowpredictive relative to predictive continuations typically shows a posterior distribution. When the target word is plausible, the LPC effect shows an anterior distribution. Note that the posterior LPC effect is the effect that has been mostly reported in general language literature, associated with discourse-level repair and re-interpretation of semantically ill-constructed language (Kuperberg, 2007; Brouwer et al., 2012). The anterior LPC effect has been implicated in the frontal inhibition network suppressing competing plausible meanings (DeLong et al., 2014).

Early cortical responses to emotional words relative to neutral words have been reported in many single word studies (Citron, 2012). The early effects range from as early as $80-120$ ms (Hofmann et al., 2009a) to 200-300 ms (Kissler et al., 2007; Kanske and Kotz, 2007; Herbert et al., 2008). It has been suggested that these early effects reflect the allocation of attentional resources to the arousing dimension of the emotional words (Schacht and Sommer, 2009; Schupp et al., 2004). Late ERP effects for emotional words, such as Late Positive Potentials (LPP), are even more commonly reported (Kanske and Kotz, 2007; Schacht and Sommer, 2009; Hinojosa et al., 2010). However, the directionality of the late emotion effects varies from study to study, and is likely to be driven by tasks (Fischler and Bradley, 2006). These late effects in single word studies have been argued to reflect the valence dimension or/and the arousal dimension (Bayer et al., 2012; Recio et al., 2014).

A number of studies have examined the processing of emotional words when the words are embedded in emotionally neutral sentences that are non-predictive but sensible. Scott et al. (2012) focused on two factors of bottom-up word processing: word frequency and word valence. A word frequency by valence interaction was found in the $135-180 \mathrm{~ms}$ range. Specifically, people fixated on emotional words shorter than they did on neutral words, but only in the low-frequency conditions, not in the highfrequency conditions. The authors suggested that the emotional content increases automatic vigilance but decreases sensitization, both of which play an important role in the processing of word frequency. Holt et al. (2009) examined emotional words embedded in passages such as "Colin decided to walk to the market. On the way he saw a snake/diamond/button on the ground". Similar to Scott et al. (2012), the passages were emotionally neutral, nonpredictive, and sensible. N400 effects were found for the emotional words (snake/diamond) relative to the neutral words (button). The authors suggested that the emotional features of the emotional words lead to deeper semantic analysis. Notably, their reported N400 effect ranged from 325 to $425 \mathrm{~ms}$, different from the typical N400 time frame of $\sim 300-500 \mathrm{~ms}$. While Holt et al. (2009) used an early N400 time window to minimize its overlap with the LPC emotion effect following N400, it is possible that this negativity effect is related to the posterior negativity effects from 150 to $300 \mathrm{~ms}$ reported in emotional word and non-linguistic stimuli (Herbert et al., 2008; Kissler et al., 2007).

Delaney-Busch and Kuperberg (2013) examined emotional and neutral words embedded in emotional and neutral contexts. In the neutral context conditions (e.g., Lucy was a female engineer. Her creations were big bridges/murals every time.), the semantically incongruent words (murals) elicited more negative N400s than the congruent words (bridges), replicating the vast N400 literature. In the emotional context conditions (e.g., Lucy was an awful/great engineer. Her creations were big failures/successes every time), emotion was manipulated via a positive word (great) and a negative word (awful). The N400 congruity effect was attenuated for emotional words, which subsequently yielded an LPC emotion effect regardless of congruity. The authors argued that the emotional contexts may have acted as a task which oriented the readers' attention toward the emotional properties of the incoming words, prioritizing emotional meaning and bypassing conceptual-semantic meaning. The authors suggested that this LPC effect may reflect the evaluation of the then focused emotional meaning.

In short, prediction accumulated in the pre-target context, when not met by the target word, elicited N400 and posterior LPC effects, which have been theorized to index semantic retrieval and semantic reanalysis, respectively. Second, the emotional content of words, when contrasted with neutral words, gave rise to early effects at $\sim 200-300 \mathrm{~ms}$ and late positivity effects, which have been suggested to reflect emotional arousal and emotional valence. Third, while N400 effects for emotion in single word studies are actually quite uncommon (Kissler et al., 2006), emotional words embedded in neutral and non-predictive (but sensible) sentences have been reported to elicit larger negativity effects than neutral words in the $325-425 \mathrm{~ms}$ time frame. The negativity effects have been suggested to reflect deeper semantic processing for emotional than for neutral words in sentences. Finally, emotional words in emotional contexts/tasks, when mismatched with context emotionally, elicited a LPC effect, which has been suggested to index affective evaluation.

The present study examined emotional and neutral words in predictive and non-predictive (anomalous) sentences, to assess the contribution of emotional word meaning and contextually driven prediction of meaning during sentence processing. Based on the reviewed literature, we expected that the ERP effects associated with the contribution of emotional word meaning should emerge as an early effect before $\sim 300 \mathrm{~ms}$ and as a late positivity after $\sim 500 \mathrm{~ms}$. The ERP effects associated with the contextually driven prediction should emerge as a negativity in the 300$500 \mathrm{~ms}$ time frame and as a late positivity effect after $\sim 500 \mathrm{~ms}$. Of particular interest was when the prediction effect and the emotion effect interact. 
Table 1

Example Dutch stimuli translated to English. The target words are underlined.

\begin{tabular}{|c|c|c|}
\hline Example & Emotional target & Non-emotional target \\
\hline Predictive context & $\begin{array}{l}{[\mathrm{p}+\mathrm{e}+] \text { The book by Roald Dahl in which Charlie is the main character }} \\
\text { takes place in a factory that makes chocolate ... }\end{array}$ & $\begin{array}{l}\text { [p+e-] The tale of Rapunzel is about Rapunzel who was held in a } \\
\text { high tower by the witch ... }\end{array}$ \\
\hline Non-predictive context & $\begin{array}{l}\text { [p-e }+ \text { ] The tale of Rapunzel is about Rapunzel who was held in a high } \\
\text { chocolate by the witch ... }\end{array}$ & $\begin{array}{l}\text { [p-e-] The book by Roald Dahl in which Charlie is the main character } \\
\text { takes place in a factory that makes tower ... }\end{array}$ \\
\hline
\end{tabular}

\section{Material and methods}

\subsection{Participants}

Thirty-three right-handed native Dutch speakers at the Radboud University Nijmegen participated in the experiment for payment. All had normal or correctedto-normal vision, and none had any language disorder, neurological disorder, or major head injury diagnosed to have long-term side effects. All gave informed consent before participation. Data were discarded from 5 participants: 4 due to technical failures and 1 due to blinking artifacts. The remaining twenty-eight participants (4 male, average age 21.2 , range $18-27$ ) were included in the analysis.

\subsection{Materials and design}

A $2 \times 2$ factorial design was employed: 2 pre-target sentential context predictability (predictive, non-predictive) x 2 target word arousal (emotional, nonemotional). This results in 4 conditions: $[\mathrm{p}+\mathrm{e}+]$ predictive context and emotional target, $[\mathrm{p}+\mathrm{e}-]$ predictive context and non-emotional target, $[\mathrm{p}-\mathrm{e}+]$ non-predictive context and emotional target, and [p-e-] non-predictive context and non-emotional target. Examples are available in Table 1 and full materials, Supplementary materials.

The materials used in the eventual EEG experiment consisted of 53 quadruplets (212 sentences), which were created and selected based on the following steps: First, approximately 100 pairs of emotional words and neutral words were selected as the target words from a Dutch affective norm (Moors et al., 2013), such that the emotional and neutral words did not differ in terms of concreteness, log frequency, word length, and part of speech (Table 2). Next, three native-speaking Dutch research assistants created the pre-target sentential contexts for each target word, such that the context was highly predictive of its word, and that the contexts between the two target words within a pair are similar in terms of valence, arousal, concreteness, expectedness parameterized using cloze probabilities here, ${ }^{2}$ length, and syntactic structure (Table 2). The non-predictive conditions were created by swapping the contexts between the two target words within a pair. Thus 100 quadruplets (400 sentences) were created at this stage of the stimuli construction.

To select from the 100 quadruplets and also to verify the designed properties of the selected quadruplets, two pretests were carried out. First, in the cloze test, 12 participants who did not participate in the EEG experiment were randomly presented with each pre-target sentential fragment and were asked to write down the first word that came to mind as the best continuation of that fragment. The cloze probability for each pre-target sentential fragment was calculated by dividing the "experimenter-expected" responses, i.e., the pre-designed target words, by the total number of responses. Second, in the emotional and concreteness test, 13 participants who did not participate in the cloze test or the EEG experiment were

\section{Table 2}

Means and standard deviations of pretest ratings. "Emotional" refers to pre-target contexts followed by emotional target words and "Neutral" refers to pre-target contexts followed by neutral target words. "Val-Arousal" refers to valence ratings on a 1-9 scale re-calculated based on distance from the center of the scale (i.e., 5), which essentially represents another way of parameterizing arousal-ness. "**” means significant difference between the listed means.

\begin{tabular}{llllll}
\hline $\begin{array}{l}\text { Pre-target } \\
\text { contexts }\end{array}$ & Val-Arousal & Arousal & Concreteness & Cloze prob. & Length \\
\hline $\begin{array}{l}\text { “Emotional" } \\
\text { “Neutral" }\end{array}$ & $1.4(1.0)$ & $5.1(1.2)$ & $6.3(0.7)$ & $0.88(0.1)$ & $68.2(11.7)$ \\
& $1.7(0.8)$ & $5.3(1.3)$ & $6.3(0.7)$ & $0.87(0.2)$ & $71.8(13.4)$ \\
$\begin{array}{c}\text { Target } \\
\text { words }\end{array}$ & Val-Arousal* & Arousal* & Concreteness & Log freq. & Length \\
\hline $\begin{array}{c}\text { Emotional } \\
\text { words }\end{array}$ & $3.4(0.4)$ & $5.8(1.5)$ & $6.9(1.3)$ & $1.4(0.6)$ & $6.9(2.2)$ \\
$\begin{array}{c}\text { Neutral } \\
\text { words }\end{array}$ & $0.6(0.4)$ & $4.3(1.5)$ & $7.2(1.4)$ & $1.3(0.7)$ & $6.5(1.9)$ \\
\hline
\end{tabular}

presented with each pre-target sentential fragment and were asked to rate the sentence fragment on a $1-9$ emotional valence scale $(1=$ negative; $9=$ positive), on a 1-9 arousal scale ( $1=$ exciting, active; $9=$ calm, passive), and on a $1-9$ concreteness scale $(1=$ concrete; $9=$ abstract). Based on the pretest ratings, 53 quadruplets were selected, such that the mean cloze probabilities, valence ratings, arousal ratings, and concreteness ratings were matched between the emotional and neutra ones. The ratings for the selected 53 quadruplets are summarized in Table 2 . In particular, the valence ratings were re-conceptualized as distance from the neutra (5 on the 1-9 scale), termed 'Val-Arousal', and were calculated by subtracting 5 from the raw valence ratings. This essentially provided another way of parameterizing how arousing (negative or positive) an emotional item is from neutral. Confirming our manipulations, both of the arousal dimensions for target words were significantly different $(p<.0001)$, and neither of the arousal dimensions for the pre-target contexts was different (n.s.). The quadruplets were then put into 4 lists via Latin Square rotation, and items within a list were randomized. All EEG participants saw all 4 lists but in different orders. List order is counterbalanced with participant number.

In addition to the sentences we also created comprehension questions for each of the sentences, to ensure participation. The comprehension questions were yes/ no questions, and were never focused on the target words.

\subsection{Procedure}

Each session started with a 30-min EEG setup. During the setup, participants filled out the Edinburgh Inventory of Handedness. After the setup, participants entered an electrically shielded, soundproof, and dimly lit room. They sat in a comfortable chair at a desk looking at a computer screen about $70-80 \mathrm{~cm}$ away from their eyes, resulting in a visual field range between $1^{\circ}$ and $2^{\circ}$ depending on the word length but not bigger than $2^{\circ}$.

Participants did passive reading while a sentence was presented to them word by word in each trial on the computer screen, using the software Presentation. The words were white on a black background, in Arial font, 20-point font size, and in sentence-case. Each word was presented for a length-dependent duration based on the following formula: If a word has fewer than 8 letters, multiply the number by 27 millisecond (ms) plus $187 \mathrm{~ms}$. If a word has more than 8 letters, use the duration for 8 letters. The Inter-word Interval was a black screen presented for $150 \mathrm{~ms}$. The last word was presented with a period, followed by the Inter-trial Interval (ITI) of $1 \mathrm{~s}$. At the end of a trial, the participant needed to press a button to continue to the next trial.

A comprehension question was presented after $25 \%$ of the trials. For each participant, the set of trials to be followed by a comprehension question were randomly selected. The comprehension appeared after the ITI, and remained on the screen until the participant answered the question with a button press (Yes/No).

Participants were instructed to refrain from blinking and moving during word presentation, but were encouraged to blink or rest their eyes between trials and during the comprehension questions. 8 practice trials were given prior to the formal experiment. Each EEG session lasted approximately 1.5-2.0 h.

\subsection{EEG recording and analyses}

Continuous EEG was recorded from 60 surface active electrodes placed in an elastic cap (Acticap, Brain Products, Germany) arranged in an equidistant montage (Fig. 1). The left mastoid electrode served as the reference, and a forehead electrode served as the ground. A supra- to suborbital bipolar montage was used to monitor vertical eye movements (i.e., 53 and VEOG), while a right to left canthal bipolar montage was used to monitor horizontal eye movements (i.e., 57 and 25). Impedances were maintained below $5 \mathrm{k} \Omega$. EEG was amplified and digitized at $500 \mathrm{~Hz}$ with a $100 \mathrm{~Hz}$ high cut-off filter and a $10 \mathrm{~s}$ time constant $(0.1-100 \mathrm{~Hz}$ band-pass).

Brain Vision Analyzer 2.0 was used to process the EEG data. The EEG data were re-referenced off-line to the average of both mastoids, and low-pass filtered at

\footnotetext{
${ }^{2}$ There are known caveats for parameterizing prediction with cloze probability measure. Some of the caveats are that it is not sensitive to non-lexical expectancy such as thematic roles, it is not very good at measuring low lexical expectancy, and it cannot distinguish two words with a cloze of zero, which are not necessarily equally unexpected.
} 


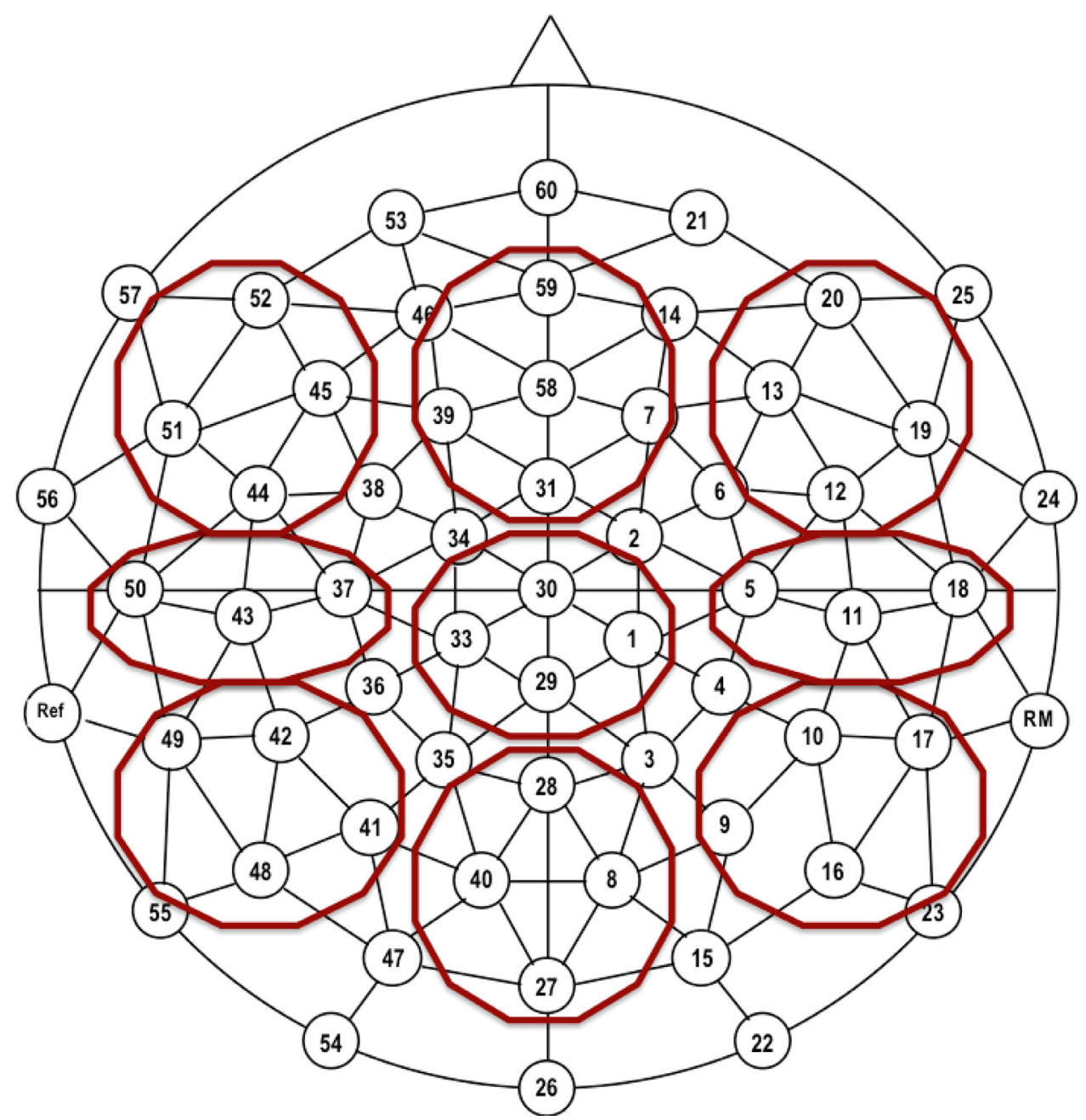

Fig. 1. 60-channel scalp electrode array. Electrodes used in the statistical analyses are circled.

$30 \mathrm{~Hz}$ (48 dB/oct slope). Blinks were detected and corrected via Independent Component Analysis (ICA). The data were segmented from $200 \mathrm{~ms}$ before the target word onset to $1000 \mathrm{~ms}$ after, with the baseline correction from -200 to $0 \mathrm{~ms}$ preceding the word onset. Segments were rejected when they contained signals exceeding $\pm 75 \mu \mathrm{V}$, and featured a linear drift of more than $\pm 50 \mu \mathrm{V}$, beginning before the onset of the target word. On average, $10 \%$ of the trials were rejected. The accepted trials were averaged for each condition for each participant, and used for further statistical analysis.

\section{Results}

The accuracy based on the comprehension questions was $86.0 \%$ ( $\mathrm{SD}=3.8 \%$ ), indicating that our participants were indeed involved in reading. The artifact rejection rates were $4 \%$ for $[p+e+], 3 \%$ for [p+e-], $3 \%$ for [p-e+], and $4 \%$ for [p-e-], with no statistical significance difference between conditions.

The representative grand averaged ERPs from the anterior, central, and posterior groups along the midline locations are displayed in Fig. 2. Based on visual inspection, condition differences emerged at $\sim 200 \mathrm{~ms}$ : First, the three conditions with predictive and/or emotional content $[\mathrm{p}+\mathrm{e}+][\mathrm{p}+\mathrm{e}-][\mathrm{p}-\mathrm{e}+]$ gave rise to more positive P200s than the non-predictive and non-emotional condition [p-e-]. Second, the non-predicted words elicited N400s more negative than the predicted words in both of the emotional and non-emotional conditions, with the N400 effects in the emotional conditions smaller than the N400 effects in the nonemotional conditions. Third, the non-predicted words elicited LPCs more positive than the predicted words in both emotional and non-emotional conditions, with the LPC effects in the emotional conditions more widely distributed than the LPC effects in the non-emotional conditions. ${ }^{3}$

Mean amplitudes were exported from 200-280 ms, 300$500 \mathrm{~ms}$, and 550-900 ms at 9 locations including the left anterior group $(44,45,51,52)$, the left central group $(37,43,50)$, the left posterior group $(41,42,48,49)$, the midline anterior group $(7,31$, $39,58,59)$, the midline central group $(1,29,30,33)$, the midline posterior group $(8,27,28,40)$, the right anterior group $(12,13,19$, $20)$, the right central group $(5,11,18)$, and the right posterior group $(9,10,16,17)$ (Fig. 1, Table 3). Data were entered into separate Repeated-Measures ANOVAs of 2 prediction (predictive, non-predictive) $\mathrm{x} 2$ emotion (emotional, non-emotional) $\mathrm{x} 3$-acp location (anterior, central, posterior) x 3-lmr location (left, midline, right) for each time window. Significant effects were followed by pairwise comparisons. The Greenhouse and Geisser (1959) correction was applied to comparisons with more than 1 degree of freedom. In addition, to address multiple comparison issues, False Discovery Rate corrections (Benjamini and Hochberg, 1995) were applied.

\section{1. $P 200(200-280 \mathrm{~ms})$}

A repeated-measures ANOVA showed a prediction $\times$ emotion

${ }^{3}$ Note that the critical words are identical in the "expected" vs. "unexpected" conditions, but that the "emotional" vs. "neutral" contrast do not have the same benefit. 


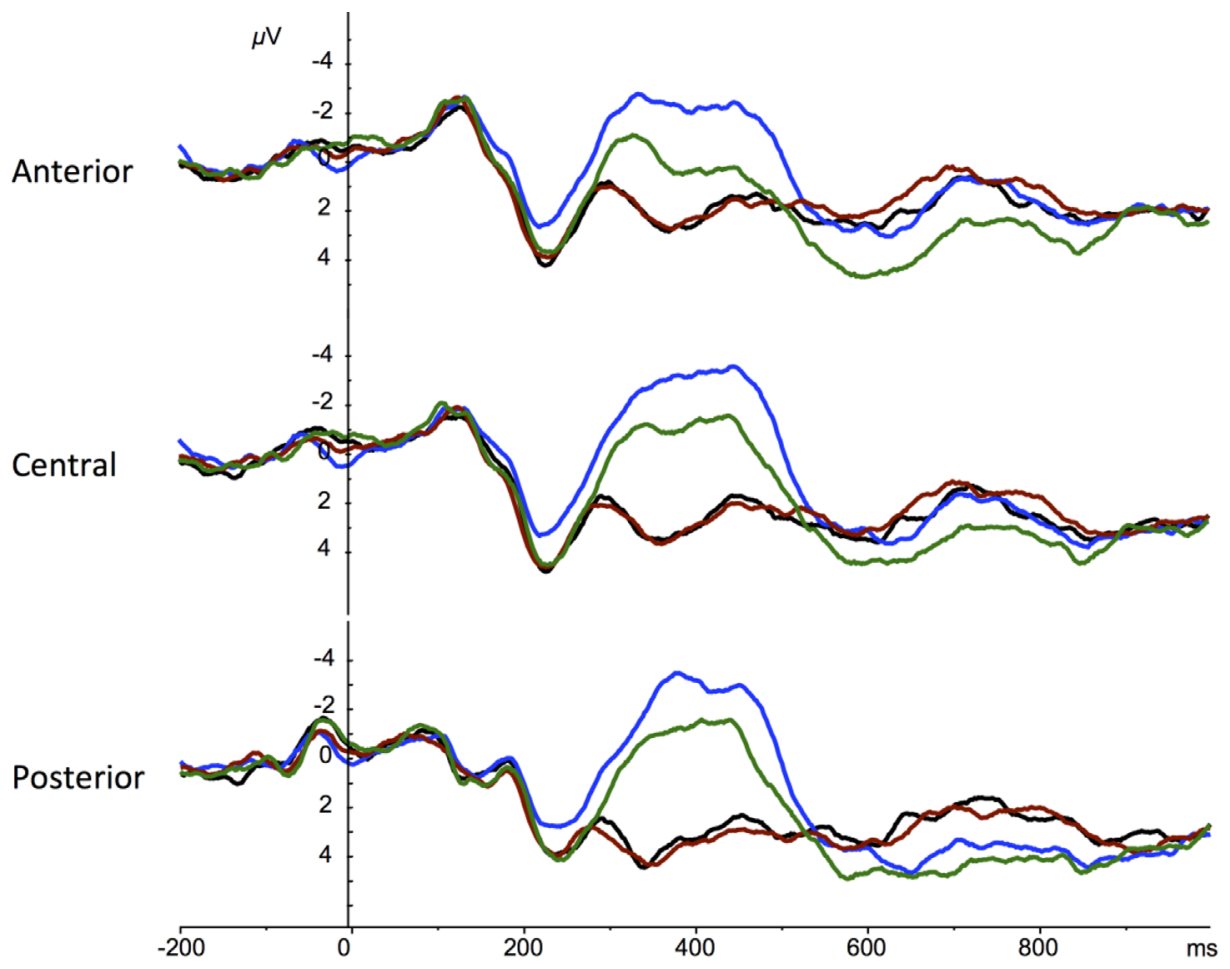

$[\mathrm{p}+\mathrm{e}+]$ The book by Roald Dahl in which Charlie is the main character takes place in a factory that makes chocolate... $[\mathrm{p}-\mathrm{e}+]$ The tale of Rapunzel is about Rapunzel who was held in a high chocolate by the witch ... [p+e-] The tale of Rapunzel is about Rapunzel who was held in a high tower by the witch ...

[p-e-] The book by Roald Dahl in which Charlie is the main character takes place in a factory that makes tower...

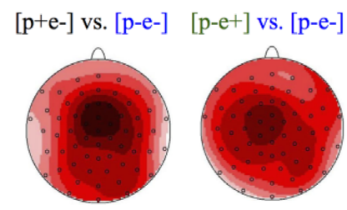

$200 \mathrm{~ms}-280 \mathrm{~ms}$

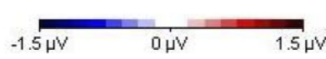

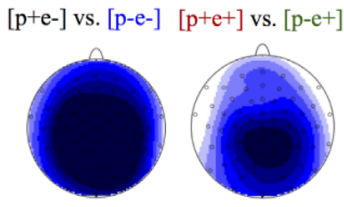

$300 \mathrm{~ms}-500 \mathrm{~ms}$

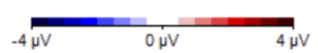

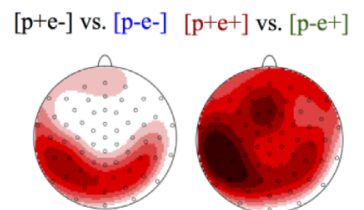

$500 \mathrm{~ms}-800 \mathrm{~ms}$

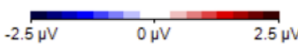

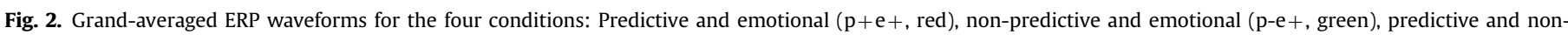

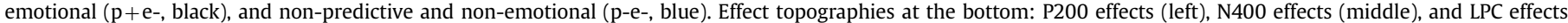
(right). (For interpretation of the references to color in this figure legend, the reader is referred to the web version of this article.)

interaction, $\mathrm{F}(1,28)=14.94, p<.001$ without location interaction, $\mathrm{F}<1$. Combining all electrode locations: Comparing the predicted and the unpredicted words when the words were non-emotional, the predicted words $[\mathrm{p}+\mathrm{e}-]$ elicited more positive P200 than the non-predicted words [p-e-], $F(1,28)=13.42, p<.001$. When the words were emotional, this prediction P200 effect disappeared, F $(1,28)=1.18, p=0.29$.

Comparing the emotional and the neutral words when the words were not predicted, the emotional words $[\mathrm{p}-\mathrm{e}+]$ elicited more positive P200 than the neutral words [p-e-], $\mathrm{F}(1,28)=14.24$, $p<0.001$. When the words were predicted, the emotion P200 effect disappeared, $\mathrm{F}(1,28)=1.57, p=0.22$.

The scalp distributions of the prediction P200 effect and the emotion P200 effect were visually similar (Fig. 2, bottom left topography). We speculated that the two effects may tax on the same neural structure(s) underlying such effect.

\section{2. $N 400(300-500 \mathrm{~ms})$}

A repeated-measures ANOVA showed a prediction $\times$ emotion interaction, $\mathrm{F}(1,28)=16.77, p<.0001$ without location interaction,
$F(1,28)=1.17, p=0.33$. Combining all electrode locations: Comparing the predicted and the unpredicted words when the words were non-emotional, the non-predicted words [p-e-] elicited more negative $\mathrm{N} 400$ than the predicted words $[\mathrm{p}+\mathrm{e}-], \mathrm{F}(1,28)=113.24$, $p<.0001$. When the words were emotional, this prediction $\mathrm{N} 400$ effect remained significant, $\mathrm{F}(1,28)=60.82, p<.0001$. The prediction $\mathrm{N} 400$ effects were more pronounced in the non-emotional compared to the emotional conditions, $\mathrm{F}(1,28)=16.77, p<.0001$ (Fig. 2, bottom middle topography), and were significant in the anterior sites, $\mathrm{F}(1,28)=18.93, p<.0001$, central sites, $\mathrm{F}(1,28)=$ $15.61, p<.0001$, and posterior sites, $\mathrm{F}(1,28)=11.01, p<.005$.

Comparing the emotional and the neutral words when the words were not predicted, the waveforms for the emotional words $[\mathrm{p}-\mathrm{e}+]$ were more negative than the neutral words [p-e-] in the $300-500 \mathrm{~ms}$ window, $\mathrm{F}(1,28)=40.09, p<0.001$. This is likely a continuation of the positivity difference at $\sim 200 \mathrm{~ms}$. When the words were predicted, the effect disappeared, $\mathrm{F}<1$.

\section{3. $L P C(550-900 \mathrm{~ms})$}

A repeated-measures ANOVA showed a prediction $\times$ emotion 
Table 3

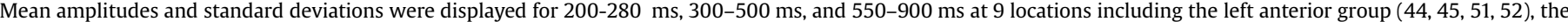

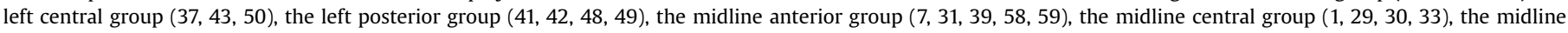
posterior group $(8,27,28,40)$, the right anterior group $(12,13,19,20)$, the right central group $(5,11,18)$, and the right posterior group $(9,10,16,17)$.

\begin{tabular}{|c|c|c|c|c|c|c|c|c|c|c|c|c|c|}
\hline & & \multicolumn{4}{|c|}{ Left Anterior Group } & \multicolumn{4}{|c|}{ Midline Anterior Group } & \multicolumn{4}{|c|}{ Right Anterior Group } \\
\hline & & $\mathbf{p}+\mathbf{e}+$ & p-e+ & $\mathbf{p}+\mathbf{e}-$ & p-e- & $\mathbf{p}+\mathbf{e}+$ & p-e + & $\mathbf{p}+\mathbf{e}-$ & p-e- & $\mathbf{p}+\mathbf{e}+$ & $\mathbf{p}-\mathbf{e}+$ & $\mathbf{p}+\mathbf{e}-$ & p-e- \\
\hline \multirow[t]{2}{*}{$200-280$} & Mean & 1.53 & 2.06 & 1.88 & 1.15 & 2.86 & 2.80 & 3.12 & 1.68 & 1.88 & 2.03 & 2.18 & 1.21 \\
\hline & Sd & 1.83 & 1.80 & 2.04 & 2.25 & 1.95 & 1.79 & 2.54 & 2.68 & 1.69 & 1.69 & 2.22 & 2.01 \\
\hline \multirow[t]{2}{*}{$300-500$} & Mean & 1.06 & 0.01 & 1.34 & 1.75 & 1.85 & 0.08 & 1.87 & 2.23 & 1.12 & 0.40 & 1.32 & 1.89 \\
\hline & $\mathrm{Sd}$ & 1.32 & 1.76 & 1.56 & 1.96 & 1.83 & 2.91 & 2.29 & 3.18 & 1.35 & 1.72 & 1.71 & 1.87 \\
\hline \multirow[t]{2}{*}{$550-900$} & Mean & 0.52 & 2.18 & 1.05 & 1.22 & 1.25 & 3.23 & 1.76 & 1.84 & 0.92 & 2.25 & 1.13 & 1.44 \\
\hline & $\mathrm{Sd}$ & 1.23 & 1.72 & 1.85 & 2.16 & 1.84 & 2.60 & 2.48 & 3.17 & 1.27 & 1.65 & 1.72 & 2.17 \\
\hline
\end{tabular}

\begin{tabular}{|c|c|c|c|c|c|c|c|c|c|c|c|c|c|}
\hline & & \multicolumn{4}{|c|}{ Left Central Group } & \multicolumn{4}{|c|}{ Midline Central Group } & \multicolumn{4}{|c|}{ Right Central Group } \\
\hline & & $\mathbf{p}+\mathbf{e}+$ & p-e + & $\mathbf{p}+\mathbf{e}-$ & p-e- & $\mathbf{p}+\mathbf{e}+$ & $\mathbf{p}-\mathbf{e}+$ & $\mathbf{p}+\mathbf{e}-$ & p-e- & $\mathbf{p}+\mathbf{e}+$ & $\mathbf{p}-\mathbf{e}+$ & $\mathbf{p}+\mathbf{e}-$ & p-e- \\
\hline \multirow[t]{2}{*}{$200-280$} & Mean & 1.42 & 2.01 & 1.76 & 1.00 & 3.41 & 3.48 & 3.55 & 2.28 & 1.91 & 2.04 & 2.10 & 1.09 \\
\hline & Sd & 1.92 & 1.85 & 2.08 & 2.56 & 2.16 & 2.02 & 2.65 & 2.68 & 1.85 & 1.53 & 2.32 & 2.14 \\
\hline \multirow[t]{2}{*}{$300-500$} & Mean & 1.71 & 0.09 & 1.97 & 1.83 & 2.84 & 0.78 & 2.75 & 2.73 & 1.96 & 0.53 & 2.02 & 2.11 \\
\hline & Sd & 1.48 & 1.74 & 1.76 & 2.41 & 2.03 & 2.64 & 2.78 & 3.36 & 1.59 & 1.70 & 2.11 & 2.12 \\
\hline \multirow[t]{2}{*}{$550-900$} & Mean & 0.71 & 3.09 & 1.24 & 2.29 & 2.36 & 4.03 & 2.59 & 2.96 & 1.64 & 3.51 & 1.66 & 2.67 \\
\hline & Sd & 1.41 & 1.81 & 1.85 & 2.36 & 1.88 & 2.93 & 2.79 & 3.42 & 1.34 & 1.92 & 2.05 & 2.56 \\
\hline
\end{tabular}

\begin{tabular}{|c|c|c|c|c|c|c|c|c|c|c|c|c|c|}
\hline & & \multicolumn{4}{|c|}{ Left Posterior Group } & \multicolumn{4}{|c|}{ Midline Posterior Group } & \multicolumn{4}{|c|}{ Right Posterior Group } \\
\hline & & $\mathbf{p}+\mathbf{e}+$ & p-e + & $\mathbf{p}+\mathbf{e}-$ & p-e- & $\mathbf{p}+\mathbf{e}+$ & p-e+ & $\mathbf{p}+\mathbf{e}-$ & p-e- & $\mathbf{p}+\mathbf{e}+$ & p-e + & $\mathbf{p}+\mathbf{e}-$ & p-e- \\
\hline \multirow[t]{2}{*}{$200-280$} & Mean & 0.96 & 1.43 & 1.32 & 0.58 & 2.80 & 3.06 & 3.11 & 2.22 & 1.79 & 1.91 & 1.91 & 1.01 \\
\hline & Sd & 2.15 & 1.98 & 2.02 & 2.40 & 2.04 & 2.19 & 2.26 & 2.29 & 1.93 & 1.67 & 2.03 & 2.06 \\
\hline \multirow[t]{2}{*}{$300-500$} & Mean & 2.53 & 0.35 & 2.60 & 1.21 & 3.25 & 0.19 & 3.11 & 1.64 & 2.78 & 0.12 & 2.66 & 1.54 \\
\hline & Sd & 1.71 & 2.12 & 1.77 & 2.70 & 1.95 & 2.74 & 2.52 & 3.45 & 1.69 & 1.88 & 2.01 & 2.24 \\
\hline \multirow[t]{2}{*}{ 550-900 } & Mean & 0.74 & 3.45 & 0.98 & 2.90 & 2.25 & 4.17 & 2.29 & 3.85 & 1.88 & 3.90 & 1.74 & 3.35 \\
\hline & Sd & 1.78 & 2.09 & 2.04 & 2.44 & 2.12 & 2.85 & 2.25 & 3.15 & 1.45 & 2.16 & 1.95 & 2.66 \\
\hline
\end{tabular}

interaction, $\mathrm{F}(1,28)=5.91, p<.05$, with a location interaction of prediction $\times$ emotion $\times$ left_midline_right $\times$ anterior_central_ posterior, $\mathrm{F}(1,28)=3.93, p<0.05$.

When the words were not emotionally valenced, the nonpredicted words elicited more positive LPCs than the predicted words, $\mathrm{F}(1,28)=4.95, p<.05$. This significance was primarily driven by the posterior site $[\mathrm{F}(1,28)=15.37, p<.001]$ and somewhat the central sites, $\mathrm{F}(1,28)=4.93, p<.05$, but not the anterior site, $\mathrm{F}<1$. When the words were emotionally valenced, the nonpredicted words elicited more positive LPCs than the predicted words, $\mathrm{F}(1,28)=38.74, p<.0001$, at the anterior $\mathrm{F}(1,28)=31.66$, $p<.0001$, central $\mathrm{F}(1,28)=36.75, p<.0001$, and posterior $\mathrm{F}(1,28)=$ 35.07, $p<.0001$, locations.

\section{Discussion}

Current research on language prediction has mostly concentrated on the processing of sentential context. The present study examined the contribution of emotional word meaning that is not prominent in the context. Participants read emotional and neutral words embedded in predictive and non-predictive sentential contexts. The crucial manipulation was that the emotional meaning was primarily activated by the target words and not from sentence context. The main finding is that both the contextual prediction effects and the emotional word effects emerged at $\sim 200 \mathrm{~ms}$. The two effects were similar in terms of time course, amplitude, and scalp distribution, suggesting potentially similar neural processes. We conjecture that such neural processes may be associated with affect and/or attention.

Prediction effects emerged at $\sim 200 \mathrm{~ms}$, more positive for confirmed than for violated predictions. One can reasonably argue that this P200 prediction effect is but an early onset of the following N400. While we acknowledge this possibility, we argue that the current data present a case where the P200 may be distinguishable from the $\mathrm{N} 400$, as the P200 effect for confirmed prediction was present in the non-emotional conditions, but was absent/eliminated in the emotional conditions, whereas the N400 effect remained in both non-emotional and emotional conditions. In addition, several other studies reported similar P200 effects for confirmed predictions (Federmeier et al., 2005; Molinaro and Carreiras, 2010; Lau et al., 2013; Chou et al., 2014). Federmeier et al. (2005) found that strongly constrained endings elicited a positivity more positive than weakly constrained endings peaking at $225 \mathrm{~ms}$ in the right visual field / processed by the left hemisphere. Lau et al. (2013) examined related and unrelated word pairs in high-predictive and low-predictive experimental contexts, and found that related words elicited an early positivity effect more positive than the unrelated words from 205 to $240 \mathrm{~ms}$ in the high-predictive context. Molinaro and Carreiras (2010) examined idioms that have predictive endings, and found an early positivity from 250 to $350 \mathrm{~ms}$ more positive for idiomatic (predictable) endings than for control (non-predictable) endings. Chou et al. (2014) examined classifier-noun pairs (e.g., a loaf of bread) in which the nouns following the classifier are highly predictable. Correct noun endings elicited more positive P200s than incorrect nouns.

How might the P200 be related to prediction and emotion processing? One possibility is that confirmed predictions lead to something akin to an "aha moment". A number of ERP studies examining the issue of insight problem solving found that problem solvers showed a positivity starting at $\sim 200 \mathrm{~ms}$ more positive than non-solvers (Lang et al., 2006; Qiu et al., 2008). Moreover, several ERP studies examining the processing of reward observed a 
P200 that was more positive for reward than non-reward conditions (Parvaz et al., 2012). Here, an affective process similar to insight and reward may occur when predictions are met. In other words, language prediction, when fulfilled, may induce an "aha moment" that feels rewarding, leading to the same positivity effect observed in the current study.

Another possibility is that both prediction and emotion tapped into the same function, namely, attentional processes. Research in the field of attention has reported a family of attention-related ERP components, including the P1, N1, P2, and P300 (Luck et al., 2000). Other studies also showed that relevant cues elicited attentionrelated P200s (Carretié et al., 2001). Moreover, single word studies of emotion typically found early emotion effects and suggested that the early effects reflect the allocation of attentional resources (cf. Introduction). Our results are consistent with the idea that both predicted words and emotional words engage attention rapidly (the timing of the effect), and very likely reach ceiling (the strength of the effect).

Disconfirmed prediction gave rise to N400 effects in the conditions where the target words were neutral. Because our nonpredictive conditions were essentially anomalous conditions, the N400 effect could be due to semantic violation (Kutas and Federmeier, 2011), prediction (DeLong et al., 2014), or both. Disconfirmed prediction also gave rise to LPC effects at the posterior locations in the conditions where the target words were neutral, consistent with past findings showing that the LPC effect for implausible continuations has a posterior distribution (DeLong et al., 2014).

Finally, we speculate that there may be two types of LPC effects at play, differentiated by their scalp distributions. One of the LPCs was posteriorly distributed, associated with prediction. The other LPC was anteriorly and centrally distributed, associated with emotion. According to this account there is a difference in the neuro-generators underlying the prediction and emotion effects observed on the scalp. The two types of LPC effects interacted at the anterior site $(F(1,28)=10.77, p=.003)$, suggesting that the LPC effect from the target word emotion boosted the LPC effect from the contextual prediction at the anterior site and thus resulting in an enhanced LPC effect from the contextual prediction. Our speculation, however, should be treated with caution due to the limitation of spatial resolution in EEG.

Overall, our findings suggest that both sentence context based prediction and target word based emotion influence processing of meaning rapidly. In an affective account, the ERP correlate for confirmed prediction resembles that for the effects of emotion, suggesting that fulfilled predictions may feel rewarding. In an attention account, both prediction and emotion enhance attentional processing early on. While further studies are needed to tease apart these two accounts, they suggest that the contribution of word processing to (predictive) sentence processing should not be overlooked. Comprehensive models of prediction must take such influences into account.

\section{Appendix A. Supporting information}

Supplementary data associated with this article can be found in the online version at http://dx.doi.org/10.1016/j.neuropsychologia. 2016.03.014.

\section{References}

Bayer, M., Sommer, W., Schacht, A., 2012. P1 and beyond: functional separation of multiple emotion effects in word recognition. Psychophysiology 49 (7), 959-969.

Benjamini, Y., Hochberg, Y., 1995. Controlling the false discovery rate: a practical and powerful approach to multiple testing. J. R. Stat. Soc. Ser. B (Methodol.), 289-300.

Brouwer, H., Fitz, H., Hoeks, J., 2012. Getting real about semantic illusions: rethinking the functional role of the P600 in language comprehension. Brain Res. 1446, 127-143.

Carretié, L., Mercado, F., Tapia, M., Hinojosa, J.A., 2001. Emotion, attention, and the "negativity bias", studied through event-related potentials. Int. J. Psychophysiol. 41 (1), 75-85.

Chou, C.-J. Huang H.-W. Lee, C.-L, Lee, C.-Y, 2014. Effects of semantic constraint and cloze probability on Chinese classifier-noun agreement. J. Neurolinguistics 31, 42-54, http://doi.org/10.1016/j.jneuroling.2014.06.003.

Citron, F.M., 2012. Neural correlates of written emotion word processing: a review of recent electrophysiological and hemodynamic neuroimaging studies. Brain Lang. 122 (3), 211-226.

Delaney-Busch, N., Kuperberg, G., 2013. Friendly drug-dealers and terrifying puppies: affective primacy can attenuate the N400 effect in emotional discourse contexts. Cogn. Affect. Behav. Neurosci. 13 (3), 473-490.

DeLong, K.A., Urbach, T.P., Kutas, M., 2005. Probabilistic word pre-activation during language comprehension inferred from electrical brain activity. Nat. Neurosci. 8 8), 1117-1121.

DeLong, K.A., Quante, L., Kutas, M., 2014. Predictability, plausibility, and two late ERP positivities during written sentence comprehension. Neuropsychologia 61 150-162.

Federmeier, K.D., Laszlo, S., 2009. Time for meaning: electrophysiology provides insights into the dynamics of representation and processing in semantic memory. Psychol. Learn. Motiv. 51, 1-44.

Federmeier, K.D., Mai, H., Kutas, M., 2005. Both sides get the point: Hemispheric sensitivities to sentential constraint. Mem. Cogn. 33 (5), 871-886.

Federmeier, K.D., McLennan, D.B., Ochoa, E., Kutas, M., 2002. The impact of semantic memory organization and sentence context information on spoken language processing by younger and older adults: an ERP study. Psychophysiology 39 (2), 133-146.

Fischler, I., Bradley, M., 2006. Event-related potential studies of language and emotion: words, phrases, and task effects. Prog. Brain Res. 156, 185-203.

Greenhouse, S.W., Geisser, S., 1959. On methods in the analysis of profile data. Psychometrika 24 (2), 95-112.

Herbert, C., Junghofer, M., Kissler, J., 2008a. Event related potentials to emotional adjectives during reading. Psychophysiology 45 (3), 487-498.

Hinojosa, J.A., Méndez-Bértolo, C., Pozo, M.A., 2010. Looking at emotional words is not the same as reading emotional words: behavioral and neural correlates. Psychophysiology 47 (4), 748-757.

Hofmann, M.J., Kuchinke, L., Tamm, S., Võ, M.L., Jacobs, A.M., 2009a. Affective processing within 1/10th of a second: High arousal is necessary for early facilitative processing of negative but not positive words. Cogn. Affect. Behav. Neurosci. 9 (4), 389-397.

Holt, D.J., Lynn, S.K., Kuperberg, G.R., 2009. Neurophysiological correlates of comprehending emotional meaning in context. J. Cogn. Neurosci. 21 (11), 2245-2262.

Huettig, F., 2015a. Four central questions about prediction in language processing Brain Res. 1626, 118-135.

Kanske, P., Kotz, S.A., 2007. Concreteness in emotional words: ERP evidence from a hemifield study. Brain Res. 1148, 138-148.

Kim, A., Lai, V., 2012. Rapid interactions between lexical semantic and word form analysis during word recognition in context: evidence from ERPs. J. Cogn. Neurosci. 24 (5), 1104-1112.

Kissler, J., Assadollahi, R., Herbert, C., 2006. Emotional and semantic networks in visual word processing: insights from ERP studies. Prog. Brain Res. 156, 147-183.

Kissler, J., Herbert, C., Peyk, P., Junghofer, M., 2007. Buzzwords early cortical responses to emotional words during reading. Psychol. Sci. 18 (6), 475-480.

Kuperberg, G.R., 2007. Neural mechanisms of language comprehension: challenges to syntax. Brain Res. 1146, 23-49.

Kutas, M., Federmeier, K.D., 2000. Electrophysiology reveals semantic memory use in language comprehension. Trends Cogn. Sci. 4 (12), 463-470.

Kutas, M., Federmeier, K.D., 2011. Thirty years and counting: Finding meaning in the N400 component of the event related brain potential (ERP). Annu. Rev. Psychol. 62, 621.

Kutas, M., DeLong, K.A., Smith, N.J., 2011. A look around at what lies ahead: Prediction and predictability in language processing. Predictions in the Brain: Using Our Past to Generate a Future, pp. 190-207.

Lang, S., Kanngieser, N., Jaskowski, P., Haider, H., Rose, M., Verleger, R., 2006. Precursors of insight in event-related brain potentials. J. Cogn. Neurosci. 18 2152-2166.

Lau, E.F., Holcomb, P.J., Kuperberg, G.R., 2013. Dissociating N400 effects of prediction from association in single-word contexts. J. Cogn. Neurosci. 25 (3), 484-502.

Lau, E.F., Weber, K., Gramfort, A., Hämäläinen, M.S., Kuperberg, G.R., 2014. Spatiotemporal signatures of lexical-semantic prediction. Cereb. Cortex, bhu219.

Luck, S.J., Woodman, G.F., Vogel, E.K., 2000. Event-related potential studies of attention. Trends Cogn. Sci. 4 (11), 432-440.

Molinaro, N., Carreiras, M., 2010. Electrophysiological evidence of interaction between contextual expectation and semantic integration during the processing of collocations. Biol. Psychol. 83, 176-190.

Moors, A., De Houwer, J., Hermans, D., Wanmaker, S., van Schie, K., Van Harmelen, A.-L., Brysbaert, M., 2013. Norms of valence, arousal, dominance, and age of acquisition for 4300 Dutch words. Behav. Res. Methods 45 (1), 169-177. 
Parvaz, M.A., Maloney, T., Moeller, S.J., Woicik, P.A., Alia-Klein, N., Telang, F., Goldstein, R.Z., 2012. Sensitivity to monetary reward is most severely compromised in recently abstaining cocaine addicted individuals: a cross-sectional ERP study. Psychiatry Res. 203 (1), 75-82.

Qiu, J., Li, H., Yang, D., Luo, Y., Li, Y., Wu, Z., Zhang, Q., 2008. The neural basis of insight problem solving: an event-related potential study. Brain Cogn. 68, 100-106.

Recio, G., Conrad, M., Hansen, L.B., Jacobs, A.M., 2014. On pleasure and thrill: the interplay between arousal and valence during visual word recognition. Brain Lang. 134, 34-43.

Rommers, J., Meyer, A.S., Praamstra, P., Huettig, F., 2013. The contents of predictions in sentence comprehension: activation of the shape of objects before they are referred to. Neuropsychologia 51 (3), 437-447.

Schacht, A., Sommer, W., 2009. Time course and task dependence of emotion effects in word processing. Cogn. Affect. Behav. Neurosci. 9 (1), 28-43.

Schupp, H., Cuthbert, B., Bradley, M., Hillman, C., Hamm, A., Lang, P., 2004. Brain processes in emotional perception: motivated attention. Cogn. Emot. 18 (5), 593-611.

Scott, G.G., O'Donnell, P.J., Sereno, S.C., 2012. Emotion words affect eye fixations during reading. J. Exp. Psychol. Learn. Mem. Cogn. 38 (3), 783-792.
Storbeck, J., Robinson, M.D., McCourt, M., 2006. Semantic processing precedes affect retrieval: the neurological case for cognitive primacy in visual processing. Rev. Gen. Psychol. 10, 41-55.

Van Berkum, J.J., Brown, C.M., Zwitserlood, P., Kooijman, V., Hagoort, P., 2005. Anticipating upcoming words in discourse: evidence from ERPs and reading times. J. Exp. Psychol.: Learn. Mem. Cogn. 31 (3), 443.

Van Petten, C., Luka, B.J., 2012. Prediction during language comprehension: benefits, costs, and ERP components. Int. J. Psychophysiol. 83 (2), 176-190.

Wicha, N.Y., Moreno, E.M., Kutas, M., 2003. Expecting gender: an event related brain potential study on the role of grammatical gender in comprehending a line drawing within a written sentence in Spanish. Cortex 39 (3), 483-508.

Wicha, N.Y., Moreno, E.M., Kutas, M., 2004. Anticipating words and their gender: an event-related brain potential study of semantic integration, gender expectancy, and gender agreement in Spanish sentence reading. J. Cogn. Neurosci. 16 (7), 1272-1288.

Zajonc, R., 2000. Feeling and thinking: closing the debate over the independence of affect. In: Forgas, J.P. (Ed.), Feeling and Thinking: The Role of Affect in Social Cognition. Studies in Emotion and Social Interaction. Cambridge University Press, New York, pp. 31-58. 